\title{
Bispecific anti-HER2 and CD16 single-chain antibody production prolongs the use of stem cell-like cell transplantation against HER2-overexpressing cancer
}

\author{
KAZUHIKO KASUYA ${ }^{1,2}$, MOTOHIDE SHIMAZU ${ }^{1}$, MINAKO SUZUKI ${ }^{2}$, \\ TAKAO ITOI $^{3}$, TATSUYA AOKI ${ }^{2}$ and AKIHIKO TSUCHIDA ${ }^{2}$ \\ ${ }^{1}$ Department of Digestive Surgery, Hachioji Medical Center of Tokyo Medical University; \\ Departments of ${ }^{2}$ Surgery, ${ }^{3}$ Internal Medicine, Tokyo Medical University, Tokyo, Japan
}

Received September 11, 2009; Accepted November 13, 2009

DOI: $10.3892 /$ ijmm_00000332

\begin{abstract}
Humanized antibodies vary and have certain effects, but they are expensive and require repeated administration. We developed cells which constantly express a humanized antibody, and we performed anticancer humanized antibody therapy involving cell transplantation. Genes with the same amino acid sequence as that of the variable region of trastuzumab (Herceptin) as the humanized anti-HER2 monoclonal antibody were produced by overlap-PCR and were connected to the anti-human CD16 antibody [antiHER2+anti-CD16 single-chain antibody (anti-HER2+CD16 $\mathrm{scAb})$. For transplantation, stem cell-like cells that are immunologically tolerant and do not transform into cancer [mouse embryo fibroblast cell line C3H10T1/2 (10T1/2)] were used. The antibody was incorporated into $10 \mathrm{~T} 1 / 2$ (antibody-expressing cells) using the pMX-IRES-EGFP retroviral vector. Cell supernatants and human monocytes were exposed to the human breast cancer strain HTB131 expressing HER2, and the in vitro antibody-dependent cellmediated cytotoxicity (ADCC) effects were evaluated. After the transplantation of antibody-expressing and HTB131 cells into SCID mice, human monocytes were intermittently administered, and the in vivo ADCC effects were evaluated. We found that the ex vivo dead cell rate was $15.4 \%$ for Herceptin, $5.6 \%$ for anti-HER2+CD16 scAb, $1.5 \%$ for anti$\mathrm{CD} 16 \mathrm{scAb}$, and $2.1 \%$ for the control, demonstrating the antitumor effects of anti-HER2+CD16 scAb. In an antibodyexpressing cell transplantation model, the inhibitory effects of this antibody on HTB131 cell establishment were observed. In conclusion, the establishment of breast cancer cells in the
\end{abstract}

Correspondence to: Dr Kazuhiko Kasuya, Hachioji Medical Center of Tokyo Medical University, 1163 Tatemachi, Hachioji, Tokyo 193-0998, Japan

E-mail: kasuya-k@jcom.home.ne.jp

Key words: anti-HER2, antibody, stem cell like-cell, cell transplantation, cancer peritoneum was inhibited by the transplantation of antibodyexpressing cells. Since this method requires cell transplantation only once, the drug cost may be reduced.

\section{Introduction}

The HER2/neu proto-oncogene as erbB-2 encodes a $185-\mathrm{kDa}$ type I transmembrane receptor tyrosine kinase (1). Trastuzumab (Herceptin ${ }^{\circledR}$, Chugai Pharmaceutical Co., Ltd., Tokyo, Japan) is an anti-HER2 antibody targeting HER2 (2). It binds to the juxtamembrane region of HER2, identifying this site as a target for a humanized monoclonal antibody authorized for metastatic breast cancer overexpressing HER2 by the Japanese government in 1998. As the common administration method of trastuzumab, the optimal dose is 2-4 $\mathrm{mg} / \mathrm{kg}$ once a week. The half-life of the IgG antibody in the blood is usually about 1 week. Therefore, to maintain an effect, the continuous administration of the antibody is required. However, antibody therapies are expensive and involve repeated administration for a long period. The total cost is approximately 14,400,000 yen ( 12,000 dollars) when a patient with metastatic breast cancer undergoes medical treatment for approximately 3 years, the average life expectancy period (test calculation of our facility), since a vial of trastuzumab costs 80,000 yen ( $\sim 670$ dollars) in Japan. To lower the cost, a reduction in the number of antibody administrations must take place. We, therefore, designed a transplant cell therapy which involves constant antibody development. Regarding the cells used for transplantation, long-life cells are desirable in order to produce antibodies for the longest period possible. Furthermore, it is desirable that the cells are applicable to all indivduals and that cell suppliers can mass produce them to save on production costs. Stem celllike cells satisfy these requirements and thus are favorable candidates. Since stem cell-like cells can multiply, mass production is possible for transplant use. Also, it is known that they are immunologically tolerant, and within-species cell transplantation is possible (3). A progressive effect is required to induce antibody-dependent cell-mediated cytotoxicity (ADCC) and to produce the antibody constantly. ADCC occurs when antibodies bind to antigens on tumor cells and the antibody Fc domains engage Fc receptors on immune effector 
cells $(4,5)$. Herceptin also elicits the cytotoxicity reaction of target cells by ADCC (6).

Cancer cells continue to achieve drug resistance by all means even when an effective anticancer drug is used, but resistance to the antibody does not develop as long as the surface antigen of the cancer is preserved. Therefore, continuous antibody treatment is extremely effective for the prevention of cancer recurrence. This study is a preliminary research involving 'cancer antibody treatment using human amnion stem cells'. Since a large quantity of amniotic stem cells can be separated from a discarded placenta, ethical issues do not arise. Moreover, stem cells do not cause canceration in spite of their marked differentiation and proliferation capacities (7). In this study, we developed mouse stem celllike cells which constantly produce anti-HER2 antibodies, and we investigated whether they are appropriate candidates for use in the treatment of cancer.

\section{Materials and methods}

Vectors. We developed two single-chain antibodies (scAb) using the following methods. The amino acid sequence of the anti-HER 2 single-chain antibody constructed with the complementarity-determining region of the mouse and humanized framework region [single-chain variable fragment $(\mathrm{scFv})]$ was obtained from the antibody showing HER2 protein-neutralizing activity engineered by Carter et al (2). These genes of anti-HER2 scFv were synthesized and cloned as independent segments by employing a combination of oligonucleotide synthesis and polymerase chain reaction (PCR). They were inserted into the expression cassette of a plasmid showing unique restriction sites which permitted the assembly of genes including (5' to $3^{\prime}$ ) the cytomegalovirus promoter/enhancer, an optimal Kozak sequence (GCCCC ACC), the human Igא-chain secretion signal sequence, a sequence encoding a human constant $\kappa$-domain, 6 his tag + $m y c$ tag to aid in identification, and an SV40 polyadenylation signal (anti-HER2 scAb). The amino acid sequence of antiCD16 scFv was obtained from an antibody with a variable region of the heavy chain $(\mathrm{VH})$ and that of the light chain (VL) domain sequences of NM3E2 scFv and linker of (Gly3 Ser Ala3) x4 specificity for CD16-positive cell-binding activity (7). Anti-CD16 scAb was constructed with antiCD16 scFv instead of anti-HER2 scFv. Anti-HER2+CD16 $\mathrm{ScFv}$ were connected and introduced into the plasmid as a bispecific scAb (9-14). These constructs were sequenced to demonstrate their fidelity. The number of sequences that encoded anti-HER2+CD16 scAb and anti-CD16 scAb was 1,950 and 1,594 bases, respectively. Theoretically, the antiHER2+CD16 scAb construct has the capacity to induce ADCC (Fig. 1A). These genes were subsequently excited from each plasmid and inserted into the expression cassette of the retroviral plasmid pMXc-IRES-EGFP generously provided by Kitamura et al (15). We used these plasmids to infect $4 \times 10^{5}$ phoenix cells on a 12 -well plate using FuGENE6 Transfection Reagent (Roche Applied Science, Mannheim, Germany), collected each retrovirus in the supernatant, and purified anti-HER2+CD16 scAb, anti-CD16 $\mathrm{scAb}$, and the null retroviral vector using conventional methods. The null retroviral vector is a control with an identical backbone to these retroviral vectors, but it contains no transgene (Fig. 1B).

The mouse embryo fibroblast cell line $10 \mathrm{~T} 1 / 2$ was generously provided as a mouse stem cell-like cell line by Nishikawa et al (3). 10T1/2 cells were placed in flat-bottomed 96-well culture plates at a concentration of $1 \times 10^{5}$ cells per well in Opti-MEM (Gibco, Life Technologies Japan Ltd., Tokyo, Japan) infected by anti-HER2+CD16, anti-CD16, and the null retroviral vectors, and then incubated for $24 \mathrm{~h}$ at $37^{\circ} \mathrm{C} .10 \mathrm{~T} 1 / 2$ cells were added to polybrane on the 2 nd day and incubated for $48 \mathrm{~h}$ at $37^{\circ} \mathrm{C}$. The fluorescence of $10 \mathrm{~T} 1 / 2$ cells was observed using EGFP after supernatant removal and washing with phosphate-buffered saline (PBS). The supernatants were evaluated for the presence of antibodies containing the human $\kappa$-chain constant region (C6) by Western blot analysis using a goat anti-human Igא antibodyhorseradish peroxidase (HRP) conjugate (Sigma-Aldrich, St. Louis, MO, USA). The supernatant of the null vector was used as a negative control, and purified human IgG (10 ng) (Sigma) was used as a positive control. For the evaluation of immunoreactivity against the HER 2 protein of the antiHER2+CD16 scAb expressed by the vector, recombinant HER2 protein (generously provided by Nichirei Corp., Tokyo, Japan) was probed with anti-HER2+CD16 scAb vectorinfected cell supernatant diluted to 4,000 times. Mouse IgG (10 ng) (Sigma) was used as a negative control.

Non-specific cytotoxicity of antibodies. Supernatants of $10 \mathrm{~T} 1 / 2$ cells infected by anti-HER2+CD16 scAb, anti-CD16 and the null vectors were evaluated regarding the presence of non-specific cytotoxicity that does not depend on ADCC. HTB131 cells derived from human breast cancer expressing HER2, which was purchased from the American Type Culture Collection (Manassas, VA, USA), was used. HTB131 cells were placed in flat-bottomed 96-well culture plates at a concentration of $1 \times 10^{5}$ cells per well in RPMI-1640 (Gibco) supplemented with $10 \%$ fetal calf serum (FCS, Sigma) under a humidified atmosphere containing $5 \% \mathrm{CO}_{2}$ at $37^{\circ} \mathrm{C}$ for $6 \mathrm{~h}$. After removing the medium, HTB131 cells were incubated in the supernatant containing the anti-HER2+CD16, anti-CD16, or null vectors diluted from 1 to $2^{7}$ times for 3 days. Then, the non-specific cytotoxicity of each supernatant was measured using the LDH release assay (Roche Cytotoxicity Detection Kit, Roche).

Cytotoxicity associated with ADCC. Cytotoxicity associated with the antibody was evaluated using a single-cell cytotoxicity assay (16).

Effector cells. Blood from healthy volunteers was collected. Fresh peripheral blood lymphocytes (PBLs) $(4 \mathrm{ml})$ were separated by buoyant density centrifugation on FicollPlaque Plus (3 ml) (Amersham, General Electric Healthcare Japan, Tokyo, Japan) at $400 \mathrm{x} \mathrm{g}$ for $30 \mathrm{~min}$ at $20^{\circ} \mathrm{C}$. The lymphocyte layer was obtained, washed twice in PBS and resuspended in RPMI-1640 without FCS. They were used as effector cells $\left(2 \times 10^{6}\right.$ cells $\left./ \mathrm{ml}\right)$ after confirmation of their viability to be $>90 \%$.

Target cells. Suspended HTB131 cells $\left(2 \times 10^{6}\right.$ cells $\left./ \mathrm{ml}\right)$ in RPMI-1640 without FCS were used as target cells. The target cells were added to the medium including the supernatant of 
A

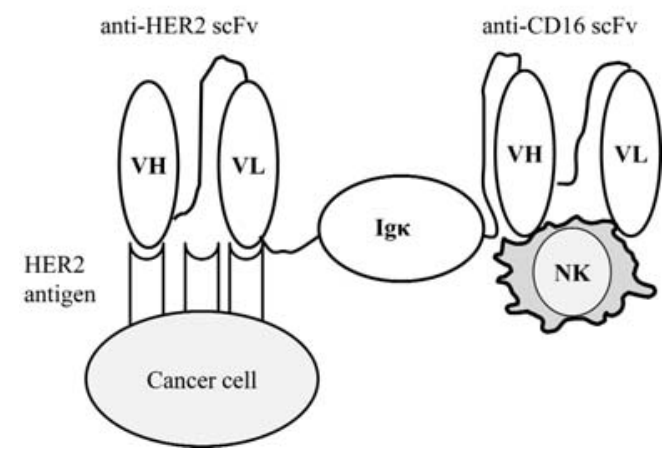

B

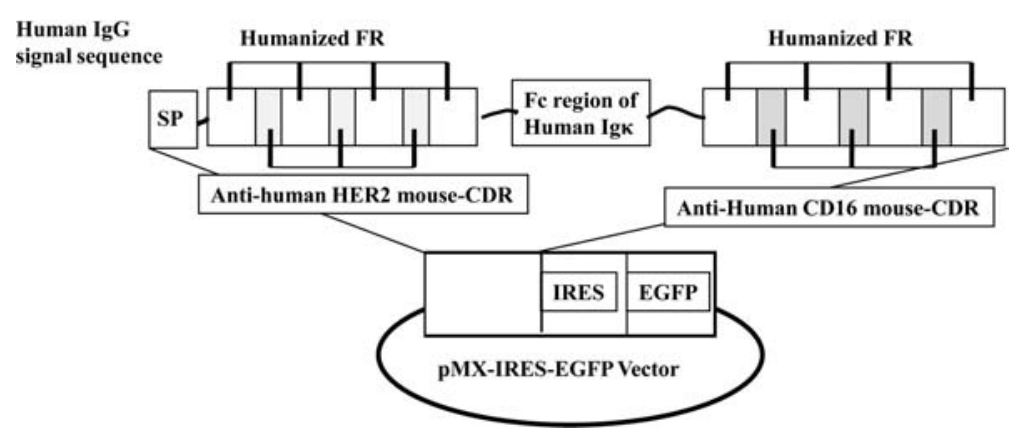

Figure 1. (A) Schematic diagram of the anti-HER2+CD16 scAb construct. Each VH and VL of anti-HER2 and those of anti-CD16 are connected via a linker (anti-HER2 scFv and anti-CD16 scFv). Anti-HER2 scFv and anti-CD16 scFv sandwich the human constant Igk domain, and are connected via a linker (antiHER2+CD16 scAb). Anti-HER2 scFv shows specificity to the extracellular protein of HER2-positive cells as a target antigen, and, also, anti-CD16 scFv shows specificity to that of CD16-positive cells such as NK cells. (B) Structure of anti-HER2+CD16 scAb retroviral vector. This retroviral vector shows IRES between the expression cassette and EGFP. VL, variable region of light chain; VH, variable region of heavy chain; NK, natural killer cell; SP, signal peptide.

anti-HER2+CD16 scAb, anti-CD16 scAb, null (no antibody) and $100 \mu \mathrm{g} / \mathrm{ml}$ of Herceptin and cultured for $72 \mathrm{~h}$ at $37^{\circ} \mathrm{C}$.

Reagents. To obtain agarose (Sigma) with optical clarity, a high-tensile strength and low melting point at $39^{\circ} \mathrm{C}$, a $1: 1$ blend of agarose and RPMI-1640 was used as molten agarose. The effector-to-target conjugates were formed using a $1: 1$ mixture of $500 \mu 1$ of effector cells and $500 \mu 1$ of target cells (final concentration of each cell type, $1 \times 10^{6}$ cells $/ \mathrm{ml}$ each) in a $10 \times 75-\mathrm{mm}$ round-bottomed borosilicate tube with subsequent centrifugation $\left(200 \mathrm{x} \mathrm{g}\right.$ ) for $5 \mathrm{~min}$ at $37^{\circ} \mathrm{C}$. Samples were incubated for $15 \mathrm{~min}$ with the subsequent addition of $1 \mathrm{ml}$ of molten agarose. The cell and agarose mixture of $200 \mu 1$ was quickly spread directly on a 6-well plate. It soon solidified, and $1 \mathrm{ml}$ of RPMI-1640 was added. It was then cultured for $3 \mathrm{~h}$ at $37^{\circ} \mathrm{C}$. After removing RPMI-1640, the gelatinous mixture of cells and agarose $(1 \mathrm{ml})$ was diluted 1:2 with $1 \mathrm{ml}$ trypan blue, left for $10 \mathrm{~min}$, washed twice with PBS and immersed in $1 \%$ formalin for $10 \mathrm{~min}$. After removing the formalin solution, the samples were washed twice with PBS and observed by microscopy. Target cells that were bound to effector cells and also stained by trypan blue were regarded as target cells killed by ADCC. The percentage of the number of dead cells to the total target cell number (500 in each well) was calculated. Four separate experiments were performed using the different procedures, and the results were calculated as the average \pm standard deviation (SD).

Double xenograft mouse. C.B-17-SCID mice (female, 4-6 weeks old, Clea Japan Inc., Tokyo, Japan) received an injection of $2 \times 10^{6}$ HTH131 cells in $0.5 \mathrm{ml}$ of RPMI-1640 medium into the peritoneal cavity (i.p.), and then an intravenous injection (i.v.) of $2 \times 10^{7}$ transgene $10 \mathrm{~T} 1 / 2$ cells via a tail vein. To assess HTH131 and 10T1/2 cell localization, mice were necropsied 6 weeks later. The HTH131 cells injected i.p. formed nodules in the intra-abdominal cavity, and the $10 \mathrm{~T} 1 / 2$ cells which were injected i.v. were implanted into the perivascular area of pulmonary arteries (double xenograft mouse). The intra-abdominal nodules and lungs were removed, fixed in formalin and embedded in paraffin.
Sections were deparaffinized in xylene, dehydrated with alcohol, rehydrated in PBS, and stained with hematoxylin and eosin (H\&E). For specimens of intra-abdominal nodules, endogenous peroxidases were blocked with $3 \%$ hydrogen peroxide in PBS. After $1 \mathrm{~h}$ of incubation with anti-ErbB2/ HER2 (C-term) rabbit monoclonal antibody (Epitomics, Inc., Burlingame, CA) diluted at 1:500, biotin-streptavidin immunostaining was carried out with CAS II Rabbit Link (Dako, Japan).

In 3 groups of double xenograft mice, 10T1/2 cells were transplanted with anti-HER2+CD16 scAb vector (antiHER2+CD16 scAb mice, $n=4$ ), anti-CD16 scAb vector (antiCD16 scAb mice, $\mathrm{n}=2$ ) and the null vector (null mice, $\mathrm{n}=2$ ). Mice were administered Herceptin at $25 \mu \mathrm{g}$ in $0.5 \mathrm{ml}$ of distilled water twice per week for 3 weeks (Herceptin mice, $\mathrm{n}=2)$. Human PBLs $\left(5 \times 10^{7}\right)$ in $0.5 \mathrm{ml}$ of PBS were injected into the peritoneal cavity of mice twice per week for 3 weeks (total number of PBL i.p. administrations was 6), and followed up for one year.

Animals were maintained at the Animal Care and Use Facilities at Tokyo Medical University Hospital under specific pathogen-free conditions. All experiments were approved by the Animal Care and Ethics Committee of Tokyo Medical University.

Statistical analysis. Statistical analyses were performed using Microsoft Excel (Microsoft Corp., Redmond, WA, USA). Differences among statistical values of single-cell cytotoxicity assays were evaluated using the Mann-Whitney Utest.

\section{Results}

In vitro characterization of the anti-HER2+CD16 scAb vector. The anti-HER2+CD16 scAb vector is a retroviral vector containing c-DNA encoding a human single-chain antibody and EGFP. To examine gene transduction and protein expression, we infected $10 \mathrm{~T} 1 / 2$ cells with anti-HER2 $+\mathrm{CD} 16 \mathrm{scAb}$ vector and observed the fluorescence of EGFP 
A

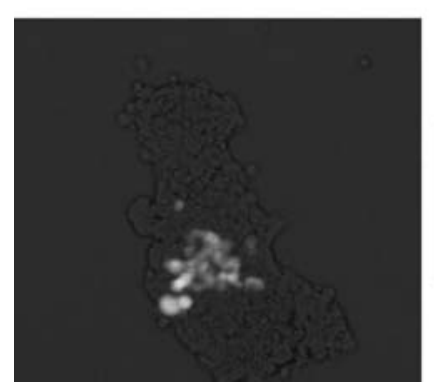

B

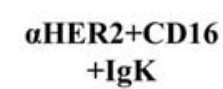

$\alpha C D 16$

(cont)
C

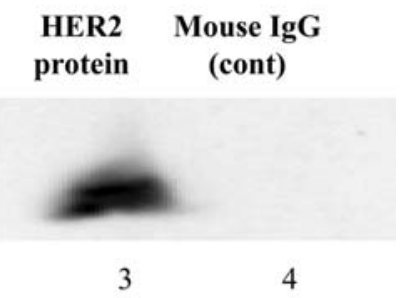

Figure 2. Expression and activity of anti-HER2+CD16 single-chain antibody using transformed 10T1/2 cells in vitro. (A) The fluorescent color development of EGFP is confirmed in the cells. (B) The expression of single-chain antibodies detected by an anti-human $\kappa$-chain (C6) antibody. Lane 1, supernatant from anti-HER2+CD16 scAb-infected cells; lane 2, anti-CD16 scAb-infected cells as a control. (C) Anti-HER2 specificity of the anti-HER2+CD16 single-chain antibody expressed by the anti-HER2+CD16 scAb retroviral vector. Lane 3, Western blot analysis of the HER2 protein using the supernatant from antiHER2+CD16 scAb-infected cells; lane 4, mouse IgG protein as a control.

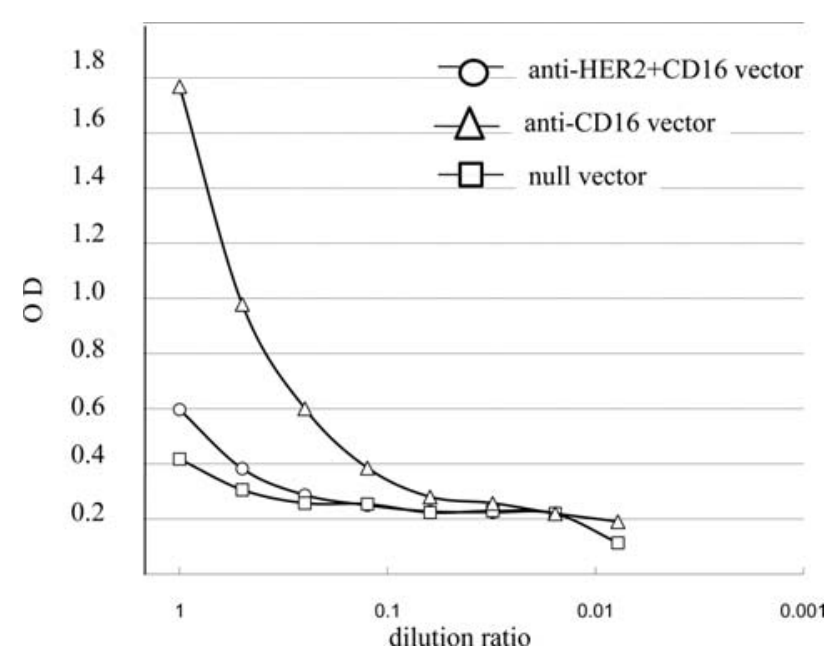

Figure. 3 Non-specific cytotoxicity of the antibodies. An LDH release assay was employed to estimate the cytotoxicity of the antibodies. The quantity of released LDH from HTH131 cells in diluted supernatants (from $2^{1}$ to $2^{8}$ ) upon infection with each retrovirus was measured. In 100-fold dilutions, no difference was observed among anti-HER2+CD16, anti-CD16, or null vectors. All data are shown as the median of 6 per group.

with a fluorescence microscope. We confirmed the stable emission of light from EGFP in the cells (Fig. 2A). To examine the expression and secretion of anti-HER2+CD16 scAb by the anti-HER2+CD16 scAb vector, anti-human human constant $\kappa$ domain, Western blot analysis under reducing conditions was performed. The supernatants collected from $10 \mathrm{~T} 1 / 2$ cells infected with the anti-HER $2+\mathrm{CD} 16$ vector contained antibodies reactive with the common human Igא chain domain expressed by the relevant retroviral vector. In contrast, no anti-human Igא-reactive material was detectable in supernatants from the null vector-infected cells (Fig. 2B). To test whether the proteins of anti-HER2+CD16 scAb bind to their respective targets, the HER2 protein, Western blot analysis was performed. Anti-HER2+CD16 scAb bound to the recombinant HER2 protein under reducing conditions, but not mouse IgG (Fig. 2C). Thus, both analyses demonstrated that the anti-HER $2+\mathrm{CD} 16 \mathrm{scAb}$ could be secreted outside $10 \mathrm{~T} 1 / 2$ cells and showed specificity for HER2. When we

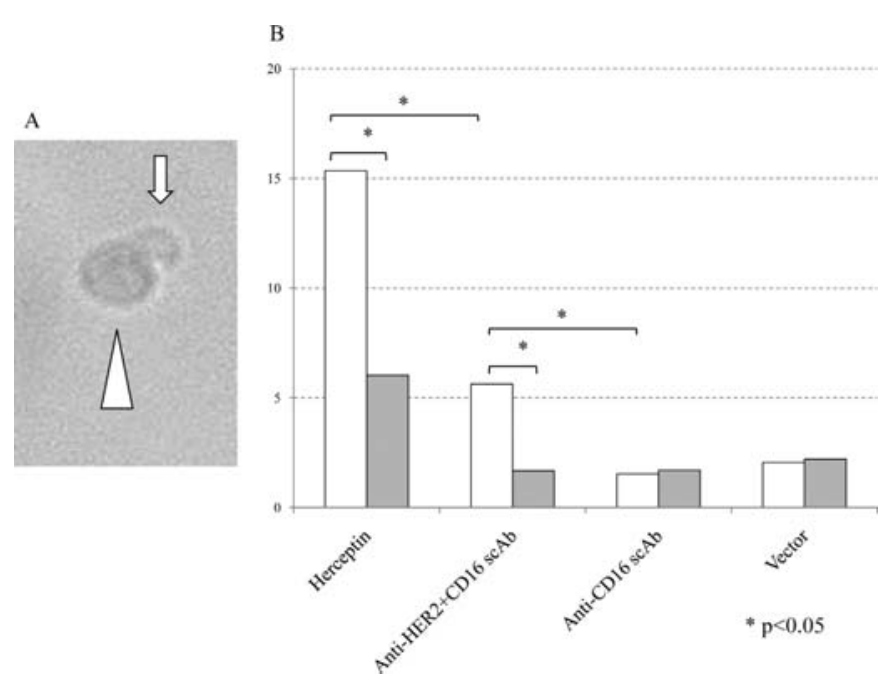

Figure 4. Single-cell cytotoxicity assay of HTH131 cells, anti-HER2+CD16 $\mathrm{scAb}$, and peripheral blood lymphocytes (PBLs). (A) Specific binding of HTH131 cells (large-sized cells, arrowhead) and PBLs (small-sized cells, arrow) is shown. Some HTH131 cells were considered to be dead cells (trypan blue-positive) due to ADCC. (B) The dead cell rate associated with ADCC is shown as a white bar, and the nonspecific dead cell rate not including mononuclear cells is shown as a gray bar. The significance of differences in the dead cell rate is shown between Herceptin and anti-CD16 $\mathrm{scAb}$, and anti-HER2+CD16 scAb and anti-CD16 scAb groups $(\mathrm{p}<0.05)$. Four separate experiments were conducted using different procedures.

investigated the presence of HER2 protein in the supernatant from the anti-CD16 vector-infected cells, anti-CD16 scAb did not recognize HER2 protein (data not shown)

Non-specific cytotoxicity of anti-HER $2+C D 16 s c A b$. We evaluated the non-specific toxicity of the supernatant including the anti-HER2+CD16 scAb, anti-CD16 scAb and null using an LDH release assay. We added supernatants from anti-HER2+CD16, anti-CD16 and null vector-infected HTH131 cells in serial dilutions. The supernatant of the cells transfected with the null vector showed the highest LDH level. Those of the anti-HER2+CD16 scAb and anti-CD16 $\mathrm{scAb}$ vectors showed values lower than the null vector in all diluted samples. However, single-chain antibodies barely 

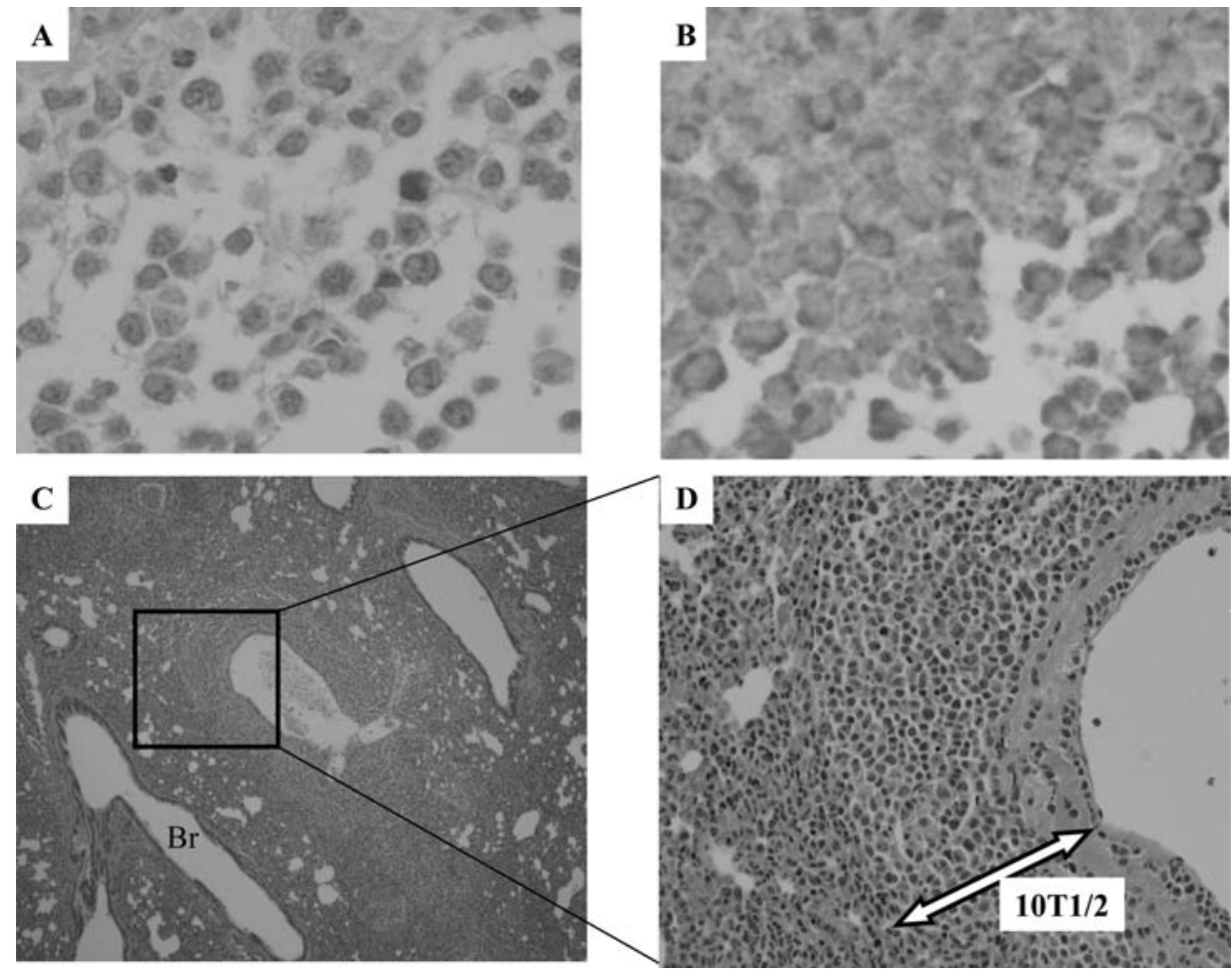

Figure 5. Histopathological findings in double xenograft mice. Upon microscopy, peritoneally disseminated nodules of HTH131 cells in the abdominal cavity were noted. (A) H\&E and (B) immunohistochemical staining with anti-human HER2 antibody. (C) In the lung, cellular groups around a pulmonary artery were observed. (D) Since such cell groups were not observed in the trachea or around the pulmonary vein, we considered these cell groups to be 10T1/2 cells administered via the tail vein. Mice showing the established peritoneal dissemination of HTH131 cells died from cancer within ten months.

showed toxicity not associated with ADCC for HTB131 HER2-positive breast cancer cells (Fig. 3).

ADCC-associated cytotoxicity of anti-HER2 $+C D 16$ scAb. We judged breast cancer cells showing trypan blue positivity connected to mononuclear cells to be ADCC-associated dead cells, and calculated a dead cell rate. The dead cell rate associated with ADCC was the highest with Herceptin $(15.4 \pm 0.5 \%)$. The dead cell rates of anti-HER $2+\mathrm{CD} 16 \mathrm{scAb}$, anti-CD16 scAb, and null were $5.6 \pm 0.5,1.5 \pm 0.2$, and $1.9 \pm 0.2 \%$, respectively. The non-specific dead cell rate of Herceptin, anti-HER2+CD16 scAb, anti-CD16 scAb, and null was $6.0 \pm 0.6,1.7 \pm 0.2,1.7 \pm 0.2$, and $2.1 \pm 0.3 \%$, respectively (Fig. 4A). Based on these results, cell death due to ADCC was caused by anti-HER2+CD16 scAb (Fig. 4B).

Antitumor effect in double xenograft mice. 10T1/2 cells were transplanted around a pulmonary artery, and there was no evidence to suggest the malignant transformation of vascular infiltrates. Small nodules were formed in the intra-abdominal cavity. These nodules were positive for HER2 protein immunohistochemically. We confirmed these implanted cells in all 4 types of mice (Fig. 5).

There was no tumor-related death in the 2 mice receiving Herceptin. One of the 4 mice undergoing $\alpha$ HER $2+\alpha C D 16$ cell administration died in the 38th week, and 2 of 2 mice receiving $\alpha \mathrm{CD} 16$ cell administration died from tumor growth in the 10th and 37th weeks (Fig. 6). An antitumor effect through cell transplantation was confirmed based on these

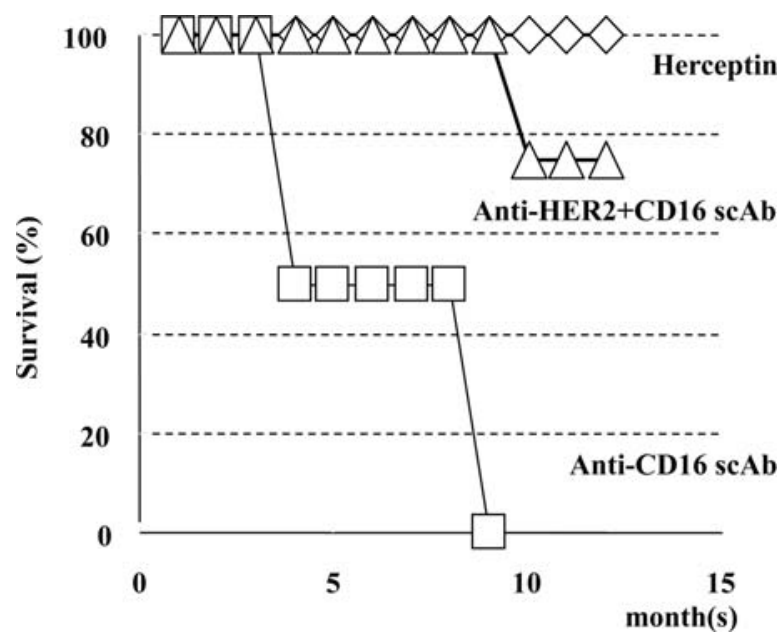

Figure 6. Survival analysis. Mice transplanted with 10T1/2 cells secreting anti-HER2+CD16 scAb $(\triangle, n=4)$, anti-CD16 scAb $(\square, n=2)$, and Herceptin $(\diamond, n=2)$ were followed up until the day of death or for one year.

results. However, the antitumor effects of the antiHER2+CD16 scAb were lower than those of Herceptin, the complete form of the antibody.

\section{Discussion}

Antibodies activate various accessory effector cells with $\mathrm{Fc}$ receptors specific to the $\mathrm{Fc}$ region of specific isotype anti- 
bodies. These accessory cells are activated after binding to specific Fc $\gamma$ receptor IgG antibodies, particularly IgG1 and IgG3 on their surface. As one population of these receptors, Fc- $\gamma$ III(CD16) receptors are present in NK cells, eosinophils and macrophages among accessory cells. The phenomenon wherein target cells bound to an antibody (breast cancer cells in this study) are destroyed by cells such as NK cells is called antibody-dependent cell-mediated cytotoxicity (ADCC) $(4,5)$. Trastuzumab and rituximab are humanized antibodies capitalizing on the effects of ADCC (6). Trastuzumab is an antibody in which the framework region and constant region have been humanized, after excluding only the complementarity-determining region directly binding to antigens (2). Of the constituent amino acids of the antibody produced in this study, 95\% were derived from human $\mathrm{IgG}$, and the other $5 \%$ were derived from the mouse antibody 4D5. Therefore, the antigenicity of the antibody in humans is very low. Its antigen affinity and specificity are comparable to those of 4D5 as the parent antibody, and ADCC induction ability is maintained. Subsequently, the antigen-binding region of trastuzumab was used in studies to enhance various antitumor effects. Weiner and colleagues studied an antiHER2+anti-CD16 single-chain antibody (8-14). They showed a decrease in CD16-positive cells only in the presence of HER2-positive target cells and trastuzumab, stating that the binding of the three is necessary. The amino acid sequence of the anti-HER2 region is the same as that of trastuzumab. In a mouse treatment model using this antibody, SK-OV-3 cells $\left(2.5 \times 10^{6}\right.$ cells $)$ as an ovarian carcinoma cell strain were administered into the peritoneum, and after 4, 6 and 8 days, IL-2-stimulated human LAK cells $\left(1 \times 10^{7}\right)$ or human PBLs and anti-HER2+anti-CD16 antibody $(100 \mu \mathrm{g})$ were intraperitoneally administered. As a result, in the untreated group, the mean survival period was 91.7-99.2 days, and no mouse survived for more than 132 days. In the treated group, the tumors did not survive for more than 150 days in $7 / 10$ mice, and the mean survival period in the other 3 mice was 128 days. Using this model, Weiner et al demonstrated the antitumor effects of the anti-HER2+CD16 antibody and human PBLs mediated by ADCC (9). The binding site of this antiCD16 antibody to CD16-positive blood cells differs from that of human IgG to these cells. Thus, this antibody has been designed so as to be able to bind to CD16 even in the presence of IgG (12-14). The basic design of anti-HER2+CD16 antibody used in this study was also originally determined based on their minibody concept.

In this study, to increase the efficiency of antitumor effects, continuous antibody expression was attempted. The degree of the effectiveness of the constant expression of antibodies, particularly single-chain antibodies, is unclear. For continuous antibody expression, Jiang et al intravenously administered adenovirus expressing an anti-HER2 singlechain antibody and reported that trastuzumab in mouse blood could be detected after day 3 , and its level reached a peak on day 7 and was maintained at $40 \mu \mathrm{g} / \mathrm{ml}$ for 4 weeks (17). The antibody directly administered was reported to be stable for $72 \mathrm{~h}$ in the blood (14). In this study, the antibody produced by $10 \mathrm{~T} 1 / 2$ cells used in vivo in mice was unable to be measured. However, we speculated that the antibody production continued due to direct gene transduction to cell genes, and the production of even a small amount of antibody activated accessory cells, inducing ADCC activity. No toxicity of the anti-HER2+anti-CD16 single-chain antibody has been previously confirmed in vitro (10) or in clinical cases (11). This study also confirmed no antibody-dependent cytotoxicity.

Studies of stem cells over the last few years have shown their potentiality as a method replacing organ transplantation. In particular, since ES cells are established from human cutaneous cells, issues regarding ethics, immune reactions at the time of transplantation, and the supply source of donor cells may be overcome in the near future. This method is also considered to be cell transplantation therapy, but its purpose is different. Amniotic cells, one type of mesenchymal stem cells (MSCs), show weak expression of MHC class I and no expression of class II, and, therefore, are difficult to recognize by the maternal immune system. These cells produce various immunosuppressive factors, inhibiting the immune system. Using these characteristics, amniotic cells are considered to be suitable for transplantation as cells not requiring HLA compatibility. In addition, since these cells are generally discarded after delivery, they are abundantly available (7). Studies on antitumor therapy using MSCs have shown MSC accumulation in the tumor $(18,19)$. A small number of MSCs were collected from the bone marrow and cultured to obtain a large amount in the laboratory. Subsequently, MSCs were transduced with an adenovirus vector encoding IFN- $\beta$ as cells for treatment. The MSCs for transplantation accumulated in the tumor, and when intravenously injected into mice, they accumulated in target cells. In addition, MSCs continuously expressed the drug (IFN-ß), resulting in cytotoxic effects on cancer cells. In gene transduction using an adenovirus vector, the gene expression period is approximately 2 weeks. Expecting gene expression for a longer period, we performed gene transduction using a retroviral vector. We considered that expression for a long period is more appropriate than high-level expression for a short period in breast cancer showing relatively slow proliferation. The gene transduction method should be determined according to the goal expression period and amount. Studeny et al $(18,19)$ scrutinized automatic MSC accumulation in the tumor rather than the antitumor effects of IFN-B. Tumors are 'injuries that do not heal', emitting various signals. MSCs receive these signals and accumulate in local areas to repair the injury. Tumors grow with the help of stem cells, arranging the cancer stromal environment. They concluded that MSC accumulation in the tumor can be an excellent vehicle for cancer treatment. Subsequently, Karnoub et al (20) reported the promotion of breast cancer metastasis by MSCs in the tumor stroma. In this study, mouse embryo fibroblast cells were used as mouse stem cell-like cells instead of MSCs, but we intend to use human amniotic cells in the future. In this study focusing on the antitumor effects of continuous antibody expression, a model in which the $10 \mathrm{~T} 1 / 2$ cell implantation area was separated from the HTH131 breast cancer cell implantation area was used. In addition, to confirm the specific cytotoxic effects of human monocytes, ADCC was demonstrated using human breast cancer cells, the humanized anti-HER2 singlechain antibody, anti-human CD16 antibody, and human monocytes. 
The most important focus in this study was the comparison of antitumor effects between the antiHER 2+CD16 antibody and Herceptin. A single-cell cytotoxicity assay for the evaluation of in vitro ADCC confirmed the cytotoxic effects of the anti-HER2+CD16 antibody due to ADCC, but its level of effect was lower than that of Herceptin. As a preliminary in vivo experiment, we produced a treatment model in which Herceptin and PBLs were administered 6 weeks after the intraperitoneal injection of tumor cells. However, no antitumor effects were observed when Herceptin and PBLs were administered after the establishment of the tumors (data not shown). These results were consistent with the absence of clinical antitumor effects of Herceptin when used alone. In this study, based on the results of the preliminary experiment on Herceptin, we did not use a model in which cells for treatment were transplanted after the establishment of HTH131 cells, but speculated that no antitumor effects can be achieved in this model. Clinically, molecular-targeted drugs are administered multiple times in principle. In this study, Herceptin was administered 6 times. Finkle et al (21) inhibited carcinogenesis using anti-HER2 antibody administration (mu4D5) in transgenic mice expressing HER2 that develop cancer generally until the 28th week, and concluded that antibody administration has inhibitory effects on carcinogenesis. Also, in this study, no tumor establishment was observed in mice receiving Herceptin. The establishment of cancer cells was also inhibited after the transplantation of cells continuously expressing anti-HER2+CD16 scAb. These results suggest the inhibitory effects of the transplantation of these cells on recurrence after an operation for breast cancer.

In conclusion, in this study, stem cells secreting the antiHER2+CD16 single-chain antibody were transplanted, and antitumor effects due to ADCC were observed. This method can be a long-term local therapy for cancer involving the single-dose administration of cells.

\section{References}

1. Bargmann CI, Hung MC and Weinberg RA: The neu oncogene encodes an epidermal growth factor receptor-related protein. Nature 319: 226-230, 1986.

2. Carter P, Presta L, Goman CM, et al: Humanization of an anti p185 (HER2) antibody for human cancer therapy. Proc Natl Acad Sci USA 89: 4285-4289, 1992.

3. Nishikawa M, Ozawa K, Tojo A, et al: Changes in hematopoiesis-supporting ability of $\mathrm{C} 3 \mathrm{H} 10 \mathrm{~T} 1 / 2$ mouse embryo fibroblasts during differentiation. Blood 81: 1184-1192, 1993.

4. Steplewshi Z, Lubeck MD and Koprowski H: Human macrophages armed with murine immunoglobulin G2a antibodies to tumors destroy human cancer cells. Science 221: 865-867, 1983.
5. Clynes RA, Towers TL, Presta LG and Ravetch JV: Inhibitory Fc receptors modulate in vivo cytoxicity against tumor targets. Nat Med 6: 443-446, 2000.

6. Brown RE, Bernath AM and Lewis GO: HER-2/neu proteinreceptor-positive breast carcinoma: an immunologic perspective. Ann Clin Lab Sci 30: 249-258, 2000.

7. Terada S, Matsumura K, Enosawa S, Miki M, Hoshika A, Suzuki S and Sakuragawa N: Including proliferation of human amniotic epithelial cells for cell therapy. Cell Transplant 9: 701-704, 2000

8. Bowles JA and Weiner GJ: CD16 polymorphisms and NK activation induced by monoclonal antibody-coated target cells. J Immunol Methods 304: 88-99, 2005.

9. Weiner LM, Holmes M, Adams GP, LaCreta F, Watts P and Palazzo IGD: A human tumor xenograft model of therapy with a bispecific monoclonal antibody targeting $c$-erbB-2 and CD16. Cancer Res 53: 94-100, 1993.

10. Weiner LM, Holmes M, Richeson A, et al: Binding and cytotoxicity characteristics of the bispecific murine monoclonal antibody 2B1. J Immunol 151: 2877-2886, 1993.

11. Weiner LM, Clark J, Davey MD, Li WS, Palazzo IGD, Ring BD and Alpaugh RK: Phase I trial of 2B1, a bispecific monoclonal antibody targeting Fc $\gamma$ RIII. Cancer Res 55: 4586-4593, 1995.

12. McCall AM, Adams GP, Amoroso AR, et al: Isolation and characterization of an anti-CD16 single chain Fv fragment and construction of an anti-HER2/neu/anti-CD16 bispecific $\mathrm{scFv}$ that triggers CD16-dependent tumor cytolysis. Mol Immunol 36: 433-446, 1999 .

13. McCall AM, Shahied LS, Amoroso AR, et al: Increasing the affinity for tumor antigen enhances bispecific antibody cytotoxicity. J Immunol 166: 6112-6117, 2001

14. Shahied LS, Tang Y, Alpaugh K, Somer R, Greenspon D and Weiner LM: Bispecific minibodies targeting HER2/neu and CD16 exhibit improved tumor lysis when placed in a divalent tumor antigen binding format. J Biol Chem 279: 53907-53914, 2004.

15. Kitamura T, Koshino Y, Shibata F, Oki T, Nakajima H, Nosaka T and Kumagai H: Retrovirus-mediated gene transfer and expression cloning: Powerful tools in function genomics. Exp Hematol 31: 1007-1014, 2003.

16. Bradley TP and Bonavida B: Mechanism of cell-mediated cytotoxicity at the single cell level. IV. Natural killing and antibody-dependent cellular cytotoxicity can be mediated by the same human effector cell as determined by the two-target conjugate assay. J Immunol 129: 2260-2265, 1982.

17. Jiang M, Shi W, Zhang Q, et al: Gene therapy using adenovirusmediated full-length anti-HER-2 antibody for HER-2 overexpression cancers. Clin Cancer Res 12: 6179-6185, 2006.

18. Studeny M, Marini FC, Dembinski JL, et al: Mesenchymal stem cells: Potential precursors for tumor stroma and targeteddelivery vehicles for anticancer agents. J Natl Cancer Inst 96: 1593-1603, 2004.

19. Studeny M, Frank C, Champlin RE, Zompetta C, Filder IJ and Andreeff M: Bone marrow-derived mesenchemal stem cells as vehicles for interferon- $\beta$ delivery into tumors. Cancer Res 62 : 3603-3608, 2002.

20. Karnoub AE, Dash AB, Vo AP, et al: Mesenchymal stem cells within tumour stroma promote breast cancer metastasis. Nature 449: 557-563, 2007.

21. Finkle D, Quan ZR, Asghari V, et al: HER2-targeted therapy reduces incidence and progression of midlife mammary tumors in female murine mammary tumor virus huHER2-transgenic mice. Clin Cancer Res 10: 2499-2511, 2004. 